\title{
UMA ATIVIDADE PIONEIRA: OS CURSOS DE EXTENSÃO EM BOTÂNICA PARA ALUNOS E PROFISSIONAIS DE MEIO AMBIENTE EM JUQUITIBA, SP
}

\author{
*Rodrigo Trassi Polisel, **Willian Fernando Domingues Vilela, ***Natália Guerin, \\ ****Rita de Cássia Sousa, ******Marcelo Del Bel
}

\section{RESUMO}

Este artigo relata uma iniciativa pioneira de alunos de graduação e pós-graduação da USP, Unicamp e Uninove, os quais vêm oferecendo, a estudantes e profissionais da área ambiental, cursos de identificação em campo das famílias botânicas arbóreas da Mata Atlântica da região Metropolitana de São Paulo em Juquitiba. O curso foi organizado com base nas coletas botânicas realizadas desde 2005 na região do Alto Juquiá. Tais coletas embasaram a produção de uma chave analítica vegetativa para reconhecimento das famílias e principais gêneros arbóreos. Do mesmo modo, foi elaborada uma apostila didática contendo glossário, chaves descritivas, técnicas de amostragem e de herborização, e um CD com fotos de mais de 220 espécies nativas regionais. Desde janeiro de 2008 foram realizados três cursos, nos quais participaram 44 alunos. Os participantes tiveram a oportunidade de observar 106 ramos de espécies nativas, agrupados em 54 famílias, proporcionando visão geral da riqueza arbórea da Mata Atlântica. Na programação do curso consta uma palestra teórica, a identificação de ramos vegetais com o uso da chave dicotômica, caminhadas na floresta e uma atividade lúdica que encerra as atividades.

Palavras-chave: Mata Atlântica. Árvores. Didática.

\section{ABSTRACT}

This article reports a pioneer initiative of a group of students from USP, Unicamp and Uninove, which have been offering a field identification course of botanical families of Atlantic Forest trees from the metropolitan region of São Paulo in Juquitiba. The course has been based on the botanical research that has been happening since 2005 in the Alto Juquiá region. This research has given material to the production of a vegetative analytical key that allows the acknowledgement of the families and main tree genders. A didactic material was also elaborated and it contains by a handbook, with glossary, descriptive keys, sampling methods and collecting, drying and pressing plants methods, as well as an illustrative CD with over 220 photos of native species. Since January 2008 three courses were offered, with 44 participants. They had the opportunity to observe 106 branches of native species belonging to 54 botanical families, providing a general idea of the richness of the tree species in the Atlantic Forest Biome. The schedule of the course includes theoretical presentation, identification of the species using a dichotomic key, walks in some forest remnants and a game that closes the activities.

Key words: Atlantic Forest. Trees. Didactic.

\footnotetext{
* Bacharel e licenciado em Ciências Biológicas pelo Instituto de Biociências da USP. Mestrando em Biologia Vegetal no Instituto de Biologia da Unicamp - e-mail: nest_usp@yahoo.com.br. ** Bacharel em Química pelo Instituto de Química de São Carlos da USP. Mestrando em Química Analítica no Instituto de Química de São Carlos da USP. *** Bacharel e licenciada em Ciências Biológicas pelo Instituto de Biociências da Unesp de Rio Claro. Mestranda em Ciências da Engenharia Ambiental da Escola de Engenharia de São Carlos da USP. ***** Bacharelanda em Ciências Biológicas pela Universidade Nove de Julho. Estagiária da Seção de Engenharia Florestal do Instituto Florestal de São Paulo. ***** Bacharel e licenciando em Ciências Biológicas pelo Instituto de Biociências da USP. Coordenador pedagógico da Empresa Caminhos \& Paisagens Viagens e Consultoria.
} 


\section{INTRODUÇÃO}

A Mata Atlântica é um dos ecossistemas mais exuberantes e potencialmente ameaçados do planeta, devido às taxas de endemismo, à riqueza biológica e ao alto grau de ameaça de seus remanescentes, sendo considerado um dos 25 hot-spots de megadiversidade do mundo (MYERS et al.,2000).

Esse bioma apresenta uma ampla variação latitudinal e altitudinal ao longo de sua área de ocorrência na costa atlântica brasileira, fatores que explicam a heterogeneidade florística e a elevada biodiversidade vegetal (TONHASCA JÚNIOR, 2005), aliado a diferentes centros de endemismos citados como blocos distintos de vegetação, como os corredores da Serra do Mar, Central da Mata Atlântica e do Nordeste (PINTO et al., 2006).

Pela complexidade do tema e falta de metodologias didáticas capazes de lidar com essa gama de informações botânicas aos estudantes universitários e profissionais afins da área de Meio Ambiente, essas informações são superficialmente trabalhadas e tornam o estudante e/ou profissional incapaz de tomar conhecimento da complexidade e exuberância do ecossistema ao final das disciplinas regulares de graduação nas instituições de nível superior.

O curso de graduação em Ciências Biológicas da USP possui duas disciplinas regulares que tratam sobre essa temática: "Diversidade e Evolução dos Organismos Fotossintetizantes" e "Taxonomia de Fanerógamas". Estas disciplinas tratam da biodiversidade vegetal como um todo e a complementação das informações aprendidas em aula acontece em visitas monitoradas ao Jardim Botânico de São Paulo de forma também superficial. Na pós-graduação, a disciplina "Taxonomia de campo: Identificação de espécies vegetais", coordenada pelo Prof. Dr. Vinícius Castro Souza, é a que realmente promove o treinamento para identificação de espécies vegetais, envolvendo as várias formas de vida, com base na coleta de ramos, preparo em laboratório e identificação de material fanerogâmico, visando à caracterização florística e sucessional das principais unidades fitogeográficas do estado.

Nessa perspectiva, torna-se inevitável a implementação de práticas de extensão universitária capazes de complementar e transmitir ainda ao aluno de ensino superior e aos profissionais da área ambiental conhecimentos sobre a composição florística dos remanescentes florestais de Mata Atlântica, possibili- tando uma visão mais técnica e menos ufanista de sua exuberância, o que praticamente só é acessível aos estudantes de pós-graduação.

A partir de 2005, levantamentos florísticos e fitossociológicos começaram a ser realizados pelos autores em trechos florestais ao longo da Bacia do Alto Juquiá, que compreende as áreas de vegetação a montante da Represa do França, em Juquitiba. Até 2008, foram levantadas 325 espécies arbóreas nativas (dados não publicados), as quais serviram para a construção da chave vegetativa de identificação das famílias e principais gêneros arbóreos regionais utilizada nesse trabalho, assim como das plantas potenciais a serem usadas como recurso didático para esse projeto.

Esse artigo tratará de uma iniciativa pioneira na área de cursos de extensão universitária em Botânica na Região Metropolitana de São Paulo proposta por alunos de graduação e pós-graduação da USP, Unicamp e Uninove, oferecidos a estudantes universitários e profissionais da área ambiental em viveiro particular de produção de mudas no município de Juquitiba. No local, é oferecido um curso para identificação em campo das famílias botânicas do estrato arbóreo da Mata Atlântica, a fim de aplicar esses conhecimentos taxonômicos na caracterização florística e fisionômica das florestas em diferentes estádios sucessionais. Além de fornecer informações úteis em métodos de levantamento da vegetação arbórea e técnicas de herborização de material botânico.

\section{MÉTODOS}

O “Curso Teórico-Prático sobre identificação em campo das principais famílias botânicas arbóreas da Mata Atlântica" possui carga horária de I6 horas (um final de semana) e é organizado e ministrado por cinco técnicos-monitores.

Cada participante recebe material didático contendo apostila com glossários, caracteres úteis na diagnose das famílias botânicas, chave de identificação, informações fitogeográficas da Mata Atlântica, técnicas de levantamento da vegetação arbórea e métodos de herborização do material botânico.

A seguir, serão descritas as atividades teóricas e práticas que cumprem o cronograma do curso: 


\section{PALESTRA DE ABERTURA (3H)}

Representa a parte teórica do curso e é apresentado na forma de powerpoint. Os conteúdos inseridos na apresentação são os seguintes:

a. Distribuição dos biomas brasileiros;

b. Delimitação do bioma Mata Atlântica através de diferentes visões na literatura;

c. As fisionomias florestais e os fatores condicionantes da Mata Atlântica;

d. A diversidade morfológica das plantas arbóreas;

e. Riqueza de espécies arbóreas na Mata Atlântica do estado de São Paulo;

f. Métodos de levantamento da vegetação arbórea.

O objetivo dessa etapa é ao mesmo tempo dar as boas-vindas aos participantes e transmitir quais serão os tópicos botânicos abordados no curso. A etapa serve também para separá-los em três grupos, os quais serão acompanhados por cada um dos técnicos-monitores na análise dos ramos frescos coletados previamente pela equipe técnica em sacos de coleta destinados ao grupo e outro, utilizado pelos monitores a fim de complementar as observações com ramos de outras espécies e grupos botânicos.

\section{OBSERVAÇÃo DE RAMOS RECÉM-COLETADOS DE ESPÉCIES ARBÓREAS NATIVAS $(6 \mathrm{H})$}

Essa é a atividade teórico-prática mais aguardada pelos participantes, pois é o momento disponível para que eles estudem os ramos de diversas famílias botânicas e aprendam quais são os caracteres morfológicos mais importantes na diagnose das famílias arbóreas e das espécies mais comuns.

Para tanto, a equipe técnica construiu uma chave de identificação vegetativa inédita para o reconhecimento das famílias arbóreas da Floresta Ombrófila Densa do Alto Juquiá (consultar os autores para o compartilhamento dessa chave), região onde o viveiro se encontra, e é a partir dessa chave que todo o estudo analítico prossegue, treinando a morfologia vegetal e aplicando-a diretamente à taxonomia.

Cada integrante coleta um ramo do saco de coleta do grupo e realiza o estudo de identificação com base na chave de identificação com o auxílio de seus colegas. Com a identificação da família conhecida, o monitor irá demonstrar possíveis dúvidas com outros materiais parecidos ou mostrar outros espécimes da família botânica identificada. Dessa forma, o saco de coleta do monitor contém ramos adicionais que auxiliarão no processo de aprendizagem e permitirão que maior número de ramos possam ser observados pelos integrantes do grupo.

Os participantes podem observar os ramos recém-coletados das espécies listadas na tabela I.

\section{TABELA 1}

Relação das 106 espécies arbóreas da região de Juquitiba coletadas e disponíveis para análise pelos participantes, organizadas por família, nome científico e nome popular (em parênteses). (G) Saco de coleta para os grupos e (M) Saco de coleta do monitor para complementação das observações.

\begin{tabular}{|c|c|c|}
\hline FAMÍLIAVESPÉCIES & $G$ & $M$ \\
\hline \multicolumn{3}{|l|}{ Anacadiaceae } \\
\hline Schinus terebinthifolius (aroeira) & I & \\
\hline Tapirira guianensis (peito-de-pombo) & & I \\
\hline \multicolumn{3}{|l|}{ Annonaceae } \\
\hline Guateria australis (araticum) & I & \\
\hline Rollinia sericea (pinha-da-mata) & & I \\
\hline Xylopia brasiliensis (pindaíba) & & I \\
\hline \multicolumn{3}{|l|}{ Apocynaceae } \\
\hline Aspidosperma olivaceum (guatambú) & I & \\
\hline Tabernamontana laeta (leiteiro) & I & \\
\hline \multicolumn{3}{|l|}{ Aquifoliaceae } \\
\hline Ilex paraguariensis (erva-mate) & I & \\
\hline \multicolumn{3}{|l|}{ Araliaceae } \\
\hline Dendropanax cuneatus (cuvantã) & & I \\
\hline Schefflera angustissima (mandioqueiro) & & I \\
\hline \multicolumn{3}{|l|}{ Asteraceae } \\
\hline Piptocarpha macropoda (vassourão) & I & \\
\hline Piptocarpha regnellii (vassourinha) & & I \\
\hline Vernonia diffusa (vassourão) & & I \\
\hline \multicolumn{3}{|l|}{ Bignoniaceae } \\
\hline Tabebuia alba (ipê-amarelo) & I & \\
\hline Jacaranda puberula (caroba) & I & \\
\hline \multicolumn{3}{|l|}{ Boraginaceae } \\
\hline Cordia sellowiana (louro-pardo) & I & \\
\hline
\end{tabular}




\begin{tabular}{|c|c|c|}
\hline FAMÍLIA & $\mathbf{G}$ & M \\
\hline \multicolumn{3}{|l|}{ Burseracea } \\
\hline Protium wildgrenii (amesca) & $\mathrm{I}$ & \\
\hline \multicolumn{3}{|l|}{ Cannabaceae } \\
\hline Trema micrantha (pau-pólvora) & $\mathrm{I}$ & \\
\hline \multicolumn{3}{|l|}{ Celastraceae } \\
\hline Maytenus robusta (cafezeiro) & I & \\
\hline \multicolumn{3}{|l|}{ Chrysobalanaceae } \\
\hline Licania hoehnei (licania) & I & \\
\hline Parinari excelsum (parinari) & & $\mathrm{I}$ \\
\hline \multicolumn{3}{|l|}{ Clethraceae } \\
\hline Clethra scabra (peroba-café) & I & \\
\hline \multicolumn{3}{|l|}{ Clusiaceae } \\
\hline Clusia criuva (clusia) & $\mathrm{I}$ & \\
\hline Garcinia gardneriana (bacopari) & & $\mathrm{I}$ \\
\hline \multicolumn{3}{|l|}{ Combretaceae } \\
\hline Buchenavia kleinii (buchenavia) & $\mathrm{I}$ & \\
\hline \multicolumn{3}{|l|}{ Cunoniaceae } \\
\hline Lamanonea ternata (guaperê) & & I \\
\hline Weinmania paullinifolia (pinheirinho) & & $\mathrm{I}$ \\
\hline \multicolumn{3}{|l|}{ Elaeocarpaceae } \\
\hline Sloanea monosperma (sapopema) & I & \\
\hline \multicolumn{3}{|l|}{ Erythroxylaceae } \\
\hline Erythroxylum argentinum (cocão) & $\mathrm{I}$ & \\
\hline \multicolumn{3}{|l|}{ Euphorbiaceae } \\
\hline Alchornea triplinervia (tapiá-mirim) & I & \\
\hline Maprounea guianensis (maprounea) & & $\mathrm{I}$ \\
\hline Sapium glandulatum (leiteiro) & & I \\
\hline \multicolumn{3}{|l|}{ Fabaceae-caesalpinoideae } \\
\hline Copaifera langsdorffii (copaíba) & I & \\
\hline Copaifera trapezifolia (copaíba-mirim) & $\mathrm{I}$ & \\
\hline Dalbergia frutescens (erva-de-rato) & I & \\
\hline Hymenaea courlbaril var. altissima (jatoba-mirim) & $\mathrm{I}$ & \\
\hline Sclerolobium denudatum (tapassuaré) & $\mathrm{I}$ & \\
\hline Senna macranthera (aleluia) & I & \\
\hline Senna multijuga (pau-cigarra) & I & \\
\hline \multicolumn{3}{|l|}{ Fabaceae-faboideae } \\
\hline Andira anthelmia (angelim) & I & \\
\hline
\end{tabular}

\begin{tabular}{|c|c|}
\hline Machaerium nictitans (jacarandá) & I \\
\hline Myrocarpus frondosus (cabreúva) & $\mathrm{I}$ \\
\hline Platymiscium floribundum (sacambu) & I \\
\hline Pterocarpus rohrii (pau-sangue) & $\mathrm{I}$ \\
\hline Ormosia dasycarpa (olho-de-cabra) & I \\
\hline \multicolumn{2}{|l|}{ Fabaceae-mimosoideae } \\
\hline Abarema langsdorffii (jacatirão) & I \\
\hline Anandenanthera colubrina (angico-preto) & I \\
\hline Inga sessilis (ingá-feijão) & $\mathrm{I}$ \\
\hline Piptadenia paniculata (serra-de-jacaré) & I \\
\hline \multicolumn{2}{|l|}{ Humiriaceae } \\
\hline Humiriastrum dentatum (humiriastrum) & I \\
\hline \multicolumn{2}{|l|}{ Lamiaceae } \\
\hline Aegiphila sellowiana (tamanqueiro) & I \\
\hline Githarexylum miryanthum (pau-viola) & I \\
\hline Vitex polygama (tarumã) & I \\
\hline \multicolumn{2}{|l|}{ Lauraceae } \\
\hline Endlicheria paniculata (canela-do-brejo) & I \\
\hline Nectandra oppositifolia (canela-amarela) & I \\
\hline Ocotea dispersa (canelinha) & $\mathrm{I}$ \\
\hline Ocotea odorifera (canela-sassafrás) & I \\
\hline \multicolumn{2}{|l|}{ Lecythidaceae } \\
\hline Cariniana estrellensis (jequitibá) & I \\
\hline \multicolumn{2}{|l|}{ Magnoliaceae } \\
\hline Talauma ovata (pinha-do-brejo) & $\mathrm{I}$ \\
\hline \multicolumn{2}{|l|}{ Malpyghiaceae } \\
\hline Byrsonima ligustrifolia (murici) & I \\
\hline \multicolumn{2}{|l|}{ Malvaceae } \\
\hline Pseudobombax grandiflorum (embiruçu) & I \\
\hline \multicolumn{2}{|l|}{ Melastomataceae } \\
\hline Miconia cabussu (cavova) & $\mathrm{I}$ \\
\hline Tibouchina pulchra (manacá) & I \\
\hline \multicolumn{2}{|l|}{ Meliaceae } \\
\hline Cabralea canjerana (canjarana) & $\mathrm{I}$ \\
\hline Cedrela fissilis (cedro-rosa) & I \\
\hline Guarea macrophyla (marinheiro) & I \\
\hline
\end{tabular}




\begin{tabular}{|c|c|}
\hline \multicolumn{2}{|l|}{ Monimiaceae } \\
\hline Mollinedia schottiana (pimenteira) & I \\
\hline \multicolumn{2}{|l|}{ Moraceae } \\
\hline Ficus luschnatiana (figueira) & I \\
\hline Sorocea bonplandii (falsa-espinheira) & I \\
\hline \multicolumn{2}{|l|}{ Myrsinaceae } \\
\hline Ardisia guianensis (capororoquinha) & I \\
\hline Rapanea umbellata (capororoca) & I \\
\hline \multicolumn{2}{|l|}{ Myrtaceae } \\
\hline Calycorectes australis (guamirim) & I \\
\hline Campomanesia phaea (cambuci) & I \\
\hline Eugenia stigmatosa (guamirim) & I \\
\hline Myrcia rostrata (guamirim-de-folha-fina) & I \\
\hline \multicolumn{2}{|l|}{ Nyctaginaceae } \\
\hline Guapira opposita (maria-mole) & $\mathrm{I}$ \\
\hline \multicolumn{2}{|l|}{ Olacaceae } \\
\hline Heisteria silvianii (chupeta-de-macaco) & I \\
\hline \multicolumn{2}{|l|}{ Oleaceae } \\
\hline Chionanthus filiformis (chionanthus) & I \\
\hline \multicolumn{2}{|l|}{ Phytollacaceae } \\
\hline Seguierea floribunda (espinheira) & I \\
\hline \multicolumn{2}{|l|}{ Piperaceae } \\
\hline Piper amalago (piper) & I \\
\hline \multicolumn{2}{|l|}{ Podocarpaceae } \\
\hline Podocarpus sellowii (pinheirinho-bravo) & I \\
\hline \multicolumn{2}{|l|}{ Polygonaceae } \\
\hline Coccoloba warmingii (coccoloba) & I \\
\hline \multicolumn{2}{|l|}{ Proteaceae } \\
\hline Roupala brasiliensis (carne-de-vaca) & I \\
\hline \multicolumn{2}{|l|}{ Quiinaceae } \\
\hline Quiina magellano-gomezii (quineira) & I \\
\hline \multicolumn{2}{|l|}{ Rosaceae } \\
\hline Prunus myrtifolia (pessegueiro-bravo) & I \\
\hline \multicolumn{2}{|l|}{ Rubiaceae } \\
\hline Alibertia myricifolia (cafezinho-do-mato) & I \\
\hline Bathysa australis (cauassu) & I \\
\hline
\end{tabular}

\begin{tabular}{|c|c|c|}
\hline Posoqueria acutifolia (laranja-de-macaco) & & I \\
\hline Rudgea jasminoides (café-do-mato) & & I \\
\hline Rutaceae & & \\
\hline Esenbeckia grandiflora (pau-de-cutia) & & I \\
\hline Zanthoxylum rhoifolium (mamica-de-porca) & I & \\
\hline Sabiaceae & & \\
\hline Meliosma sellowii (meliosma) & & I \\
\hline Salicaceae & & \\
\hline Casearia sylvestris (guaçatonga) & I & \\
\hline Xylosma glaberrima (espinho-de-judeu) & & I \\
\hline Sapindaceae & & \\
\hline Allophylus petiolulatus (chal-chal) & I & \\
\hline Cupania oblongifolia (camboatã) & I & \\
\hline Matayba intermedia (camboatá) & I & \\
\hline Sapotaceae & & \\
\hline Chrysophyllum flexuosum (falso-abiú) & I & \\
\hline Micropholis crassipedicellata (gumixava) & & I \\
\hline Pouteria bullata (guapeva) & & I \\
\hline Solanaceae & & \\
\hline Brunfelsia pauciflora (manacá-de-jardim) & & I \\
\hline Solanum swartzianum (fumo-bravo) & I & \\
\hline Symplocaceae & & \\
\hline Symplocos falcata (café-bravo) & I & \\
\hline Thymelaeaceae & & \\
\hline Daphnopsis fasciculata (pau-de-embira) & I & \\
\hline Urticaceae & & \\
\hline Cecropia pachystachya (embaúba) & I & \\
\hline Vochysiaceae & & \\
\hline Vochysia bifalcata (tucaneiro) & & I \\
\hline
\end{tabular}

\section{ATIVIDADE PRÁTICA 1: CAMINHADA NA FLORESTA $(4 \mathrm{H})$}

No dia seguinte, os três grupos percorrerão duas trilhas. A primeira irá percorrer um trecho de floresta em estágio médio de regeneração com 40 anos de idade e outro trecho de floresta em estágio avançado de regeneração, que não sofreu corte raso. O objetivo dessa caminhada é fazer os alunos discutirem quais as variações florísticas e estruturais dessas florestas, com base nas árvores jovens encontradas no sub-bosque e aquelas presentes no dossel, na estatura das árvores, 
na presença de palmeiras, cipós, epífitas e serrapilheira. Nessa trilha, os alunos terão uma visão bem fundamentada de quais são as principais observações que devem ser feitas na hora de avaliar o grau sucessional de um remanescente florestal.

A segunda trilha é percorrida concomitantemente por dois monitores e nela serão transmitidas as principais técnicas de levantamento da vegetação arbórea. Os alunos efetuarão a amostragem de um pequeno trecho de floresta a partir do uso do método de parcelas (MÜLLER-DOMBOIS et al., I974) e quadrantes (COTTAM et al., I956), além dos preceitos básicos para uma Avaliação Ecológica Rápida correta e consistente (SAYRE, 2000). Essa trilha também é utilizada para chamar atenção aos participantes dos métodos de herborização do material botânico, assim como das informações úteis a serem anotadas pelo pesquisador a fim de construir uma ficha de herbário, como estatura, observações pertinentes da planta e habitat, informações que não podem ser observadas no material seco. Ao voltarem para a propriedade, os alunos irão preparar as prensas com o material coletado e deixá-las prontas para irem à estufa.

\section{ATIVIDADE PRÁTICA 2:}

\section{CAÇA AO TESOURO BOTÂNICO (3H)}

A atividade prática final do curso mescla todos os conceitos e observações realizados tanto nas atividades de análise dos ramos, quanto nas saídas a campo. Cada grupo recebe uma dica para se encaminhar até um determinado local da propriedade onde uma árvore estará marcada e é requerida ao grupo a correta identificação. As etapas subsequentes são levadas adiante com base nos papéis corretos que são elencados pelos participantes. As outras etapas envolvem a identificação de uma muda do viveiro, sendo fornecidos os caracteres úteis da família, o encontro de uma planta de determinada família numa área previamente delimitada, o encontro de uma planta em local determinado a partir do material herborizado da mesma espécie e a identificação pelo menos até o nível de família de um grupo de plantas demarcadas num trecho de reflorestamento realizado na propriedade.

\section{RESULTADOS E DISCUSSÃO}

Três cursos foram realizados na propriedade particular em Juquitiba desde 2008. No total, foram 44 participantes: 20 no primeiro, II no segundo e I3 no terceiro. Dentre os inscritos, 28 (64\%) são profissionais da área e I6 (36\%) são estudantes.

Por restringir o curso apenas para o estrato arbóreo, os participantes podem ter uma noção mais detalhada das principais famílias botânicas que compõem esse estrato, já que no total eles terão acesso à observação de ramos de 54 famílias, que totalizam IO6 espécies. Apesar do tempo curto, há uma receptividade muito boa a esse tipo de planejamento por parte dos alunos, devido ao anseio deles em poderem observar o maior número possível de exemplares da Mata Atlântica.

Cada participante possui uma estratégia diferente em adquirir os conhecimentos, alguns anotam a maior quantidade de informações, outros preferem fotografar os caracteres ou desenhar e poucos preferem apenas prestar atenção às informações transmitidas.

É notória a falta de conhecimentos dos profissionais formados na caracterização florística e reconhecimento das espécies mais comuns tanto no campo quanto na análise dos ramos. A falta de cursos de extensão e a subjetividade das disciplinas de graduação sobre essas temáticas podem ser apontadas como razões dessa lacuna de conhecimento.

A intenção da equipe técnica é começar a incluir outras formas de vida, no estudo dos caracteres úteis para o seu reconhecimento, semelhante ao que é realizado nos principais cursos de campo de pósgraduação em Botânica, em cursos com três ou quatro dias de duração. Nesses, a ideia é que os próprios participantes possam construir uma chave analítica para reconhecer as espécies estudadas por eles, o que requererá maior nível de conhecimentos prévios dos participantes em cursos mais focados e específicos.

\section{AGRADECIMENTOS}

Os autores prestam os mais sinceros agradecimentos a todos que contribuíram para que os três cursos já realizados acontecessem da maneira excelente como foram. Alguns nomes do pessoal de apoio do viveiro Maria Tereza em Juquitiba devem ser lembrados neste espaço: Roberto Polisel, Vera Lucia Trassi Polisel, Alécio Polizel, Vanuza Vieira, Delci Vieira e Alan. Agradecemos também aos(as) proprietários(as) 
Maria de Lourdes Trassi, Sonia Maria Trassi e Wilson Souza pela autorização em utilizar os remanescentes florestais de suas propriedades.

\section{REFERÊNCIAS BIBLIOGRÁFICAS}

COTTAM, G. \& GURTIS, J. T. The use of distance measures in phytosociological sampling. Ecology, I956, v. 37 , p. 45I-46o.

MÜELLER-DOMBOIS \& ELLENBERG, H. I974. Aims and methods of vegetation ecology. New York: John Wiley \& Sons, I974.

MYERS, N.; MITTERMEIER, R. A.; MITTERMEIER, G. G.; FONSECA, G. A. B. \& KENT, J. Biodiversity hotspots for conservation priorities. Nature, 2000, v. 403, p. $853-858$.

PINTO, L. P.; BEDÊ, L.; PAESE, A.; FONSECA, M.; PAGLIA, A. \& LAMAS, I. Mata atlântica brasileira: Os desafios para a conservação da biodiversidade de um Hotspot mundial. In: ROCHA, C. F. D. et al. Biologia da conservação: Essências. São Carlos: Ed. Rima, 2006.

SAYRE, R. A natureza em foco: Avaliação Ecológica Rápida. Virgínia: Arlington, 2000.

TONHASCA JUNIOR, A. Ecologia e história natural da Mata Atlântica. Rio de Janeiro: Interciência, 2005. 